\title{
Fast Variations of Gamma-Ray Emission in Blazars
}

\author{
Stefan J. Wagner*, Corinna von Montigny, and Martin Herter \\ *swagner@lsw.uni-heidelberg.de \\ Landessternwarte, Königstuhl, 69117 Heidelberg, Germany
}

\begin{abstract}
The largest group of sources identified by EGRET are Blazars. This subclass of AGN is well known to vary in flux in all energy bands on time-scales ranging from a few minutes (in the optical and X-ray bands) up to decades (radio and optical regimes). In addition to variations of the gamma-ray flux between different viewing periods, the brightest of these sources showed a few remarkable gamma-ray flares on time-scales of about one day, confirming the extension of the "Intraday-Variability (IDV)" phenomenon into the GeV range. We present first results of a systematic approach to study fast variability with EGRET data. This statistical approach confirms the existence of IDV even during epochs when no strong flares are detected. This provides additional constraints on the site of the gamma-ray emission and allows cross-correlation analyses with light curves obtained at other frequencies even during periods of low flux.

We also find that some stronger sources have fluxes systematically above threshold even during quiescent states. Despite the low count rates this allows explicit comparisons of flare amplitudes with other energy bands.
\end{abstract}

\section{Introduction}

Most of the identified sources in the energy band studied with EGRET are Blazars. One of the defining criteria of this class is pronounced variability in the optical and radio bands on time-scales of weeks to months [13]. Later it was realized that these sources often show strong and variable X-ray emission as well. During the last decade it became clear that variations can be traced down to much shorter time-scales in all energy bands. Rapid changes "IDV" [16] can now be traced down to the shortest time-scales which can be probed in all of the different wavelength regimes. In the radio domain variations have been found to occur within less than two hours [11], [7], [5], [15], in the optical and near-IR regimes variations on timescales of hours [18], [10], [14] down to a few minutes [15] have been reported. In the X-ray regime variations have been reported to be even faster [1], [12], [18]. 


\section{S.J. WAGNER ET AL.}

Blazars also turned out to be the dominant class of sources in the $\mathrm{GeV}$ regime. It was soon realized that they vary from one EGRET viewing period to another (von Montigny, et al., this meeting). If observed during a particularly bright state, individual objects were shown to vary on shorter time-scales as well (e.g. [6]). With typical recorded flux-densities of about a photon per hour it is impossible to probe the regime of very fast variations ( $<$ hours) which can be tackled in other energy bands. Statistical approaches enable investigations of the fastest variation that can be studied. They also allow comparisons throughout the entire range of time-scales with those in other regimes of the electromagnetic spectrum.

\section{Rapid Gamma-ray Flares}

Already one of the first EGRET pointings towards a Blazar found the source 3C 279 in a state of enhanced activity. Detailed analysis revealed variations on time-scales of a few days with high significance [6], [3]. Comparable variations were found in other sources, including, e.g., PKS 0528+134 [4], PKS 1406-076 [17], PKS 1633+382 [8], and PKS 2251+158 [2]). Hunter et al. [4] illustrated that the variability of PKS $0528+134$ was persistent over at least eight weeks with several maxima rather than a singular, well defined peak. This is comparable to the variations of the synchrotron emission of Blazars in general, as seen e.g. in the optical regime [16]. In an attempt to constrain the shortest time-scales, Mattox et al. [9] analysed the brightest flare of any gamma-emitting Blazar, PKS 1633-29, by binning the light curve with a variable width in time such that the individual bins had comparable errors. This revealed time-scales as short as about 6 hours in the observers frame.

In order to study the statistical properties of fast variations we analysed the EGRET observations of a larger set of Blazars on time-scales of about one day. All observations of a selected source available from the EGRET archive were taken and binned into one-day maps. This binning was chosen to remain sensitive to the
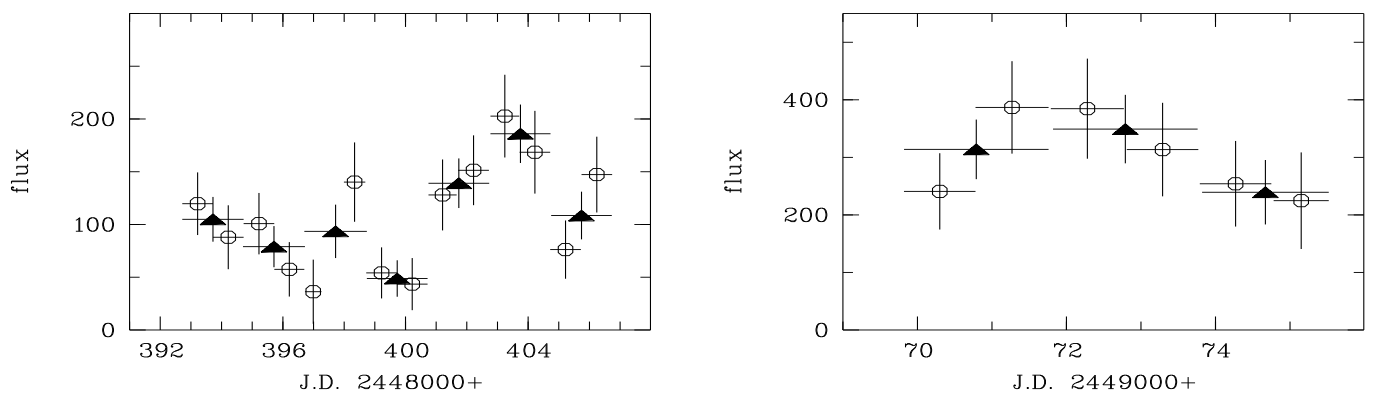

FIGURE 1. Light curves of PKS $0528+134$ with a binning of one (open circles) and two days (full triangles) during two different viewing periods (flux is given in $10^{-8} \gamma \mathrm{cm}^{-2} \mathrm{~s}^{-1}$ ). While $\chi^{2}$ tests indicate significant variations in the left panel (not in the right one), a two day binning is too poor to reveal the shortest time-scales. 


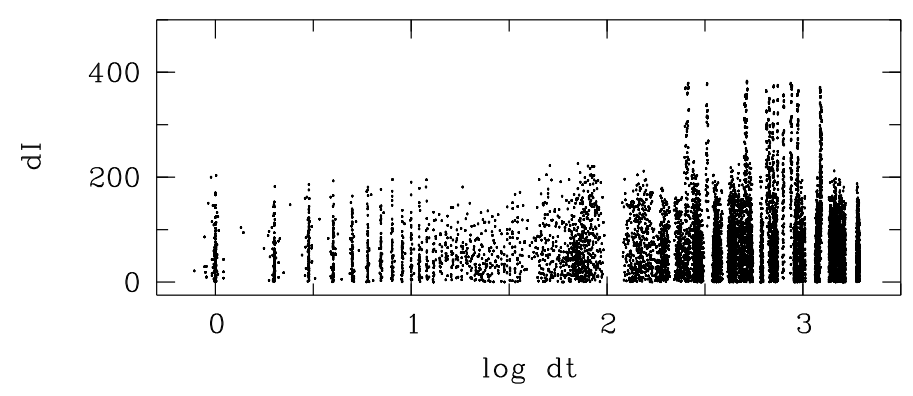

FIGURE 2. Differences in intensity of PKS $0528+134$ between any two epochs as a function of the difference in time $\log (\mathrm{dt} / 1$ day) between the measurements.

fastest changes seen in EGRET data so far. Rebinning to longer time-scales could be performed without any significant degradation in accuracy. Examples of light curves binned into intervals of one and two days, respectively, are shown in Figure 1.

The entire data set of every well-observed source can be studied in over 100 oneday maps. This is a sufficiently large data base to carry out statistical studies, comparable to the structure function analysis used in temporal studies at other wave-bands. A similar method has been used by [18] to study IDV in 0716+714 in the radio and optical bands.

\section{Statistical Analyses}

All light curves of a source, binned in one-day intervals (as shown in Figure 1 ), are combined and used to create structure functions. In order to determine characteristic temporal changes in flux, every combination of two measurements $\mathrm{I}\left(\mathrm{t}_{i}\right), \mathrm{I}\left(\mathrm{t}_{j}\right)$ is used to derive one measurement $\mathrm{dI}=\mathrm{I}\left(\mathrm{t}_{i}\right)-\mathrm{I}\left(\mathrm{t}_{j}\right)$ at $\mathrm{dt}=\left(\mathrm{t}_{i}-\mathrm{t}_{j}\right)$. The entire data set of PKS $0528+134$ is given in Figure 2. The structure function as defined by [18] was derived from the set of $\mathrm{dI}(\mathrm{dt})$ by averaging a) equal bins in $\mathrm{dt}, \mathrm{b})$ equal bins in $\log (\mathrm{dt})$, and c) bins of equal numbers of measurement points $\mathrm{dI}(\mathrm{dt})$. With the small number of measurements, the uncertainty introduced by choosing a particular binning and phase may be significant. Figure 3 illustrates the resulting structure functions, derived by using the three different ways of binning. Each panel gives four curves, representing shifts of the phase by $25 \%$ of the width of the bins.

Since the exposure of different one-day maps changed dramatically, the errors on the $\mathrm{dI}(\mathrm{dt})$ varied significantly. This is taken into account in the computation of the structure functions by individual weights but is not illustrated with error bars in Figure 2 for clarity. Instead we present another version of Figure 2 where the $\mathrm{dI}(\mathrm{dt})$ are given in terms of their significance $\mathrm{dS}(\mathrm{dt})=\mathrm{dI}(\mathrm{dt}) / \sigma_{d I}(d t)$ (Figure 4 , left panel). The right panel compares the histogram of $\mathrm{dS}(\mathrm{dt})$ for $\mathrm{dt} \sim 1$ day with the normal distribution expected for a steady source with random errors, which clearly 


\section{S.J. WAGNER ET AL.}
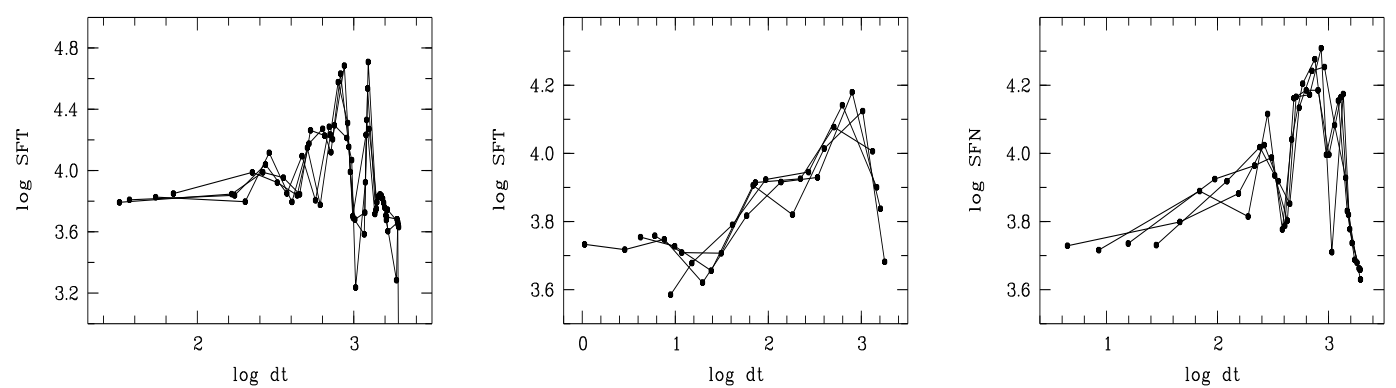

FIGURE 3. Structure functions of PKS $0528+134$ derived from bins of $\mathrm{dt}=100$ days (left panel, phase offsets of 25 days $), \log (\mathrm{dt} / 1$ day $)=0.4$ (central panel, phase offsets of $\log (\mathrm{dt} / 1$ day) $=0.1$ ), and $n=800$ (right panel, phase offset of $d n=200$ ).

illustrates the excess at large dS.

Interpreting the results shown in Figure 4 directly as evidence for the statistical occurrence of variability relies on an accurate determination and propagation of errors. We consistently chose conservative errors.

We tested different approaches by either fixing the positions of the sources or leaving them as free parameters and by fixing the background or leaving it as free parameters. Despite subtle differences, we arrived at consistent results.

As an alternative approach we treated other sources within the same pointing directions. If errors are not estimated correctly, one may still regard the least variable source within any field of view as constant and derive the actual spread in errors from the dispersion of those sources with least variability. This will allow the identification of variable sources even if there are unknown contributions to the total errors as long as constant sources exist. Although gamma-bright pulsars may be considered as being constant on time-scales much longer than their pulsation periods and much shorter than cooling time-scales, we found significant differences between the structure functions of the Crab Pulsar and Geminga. These results will be presented separately.
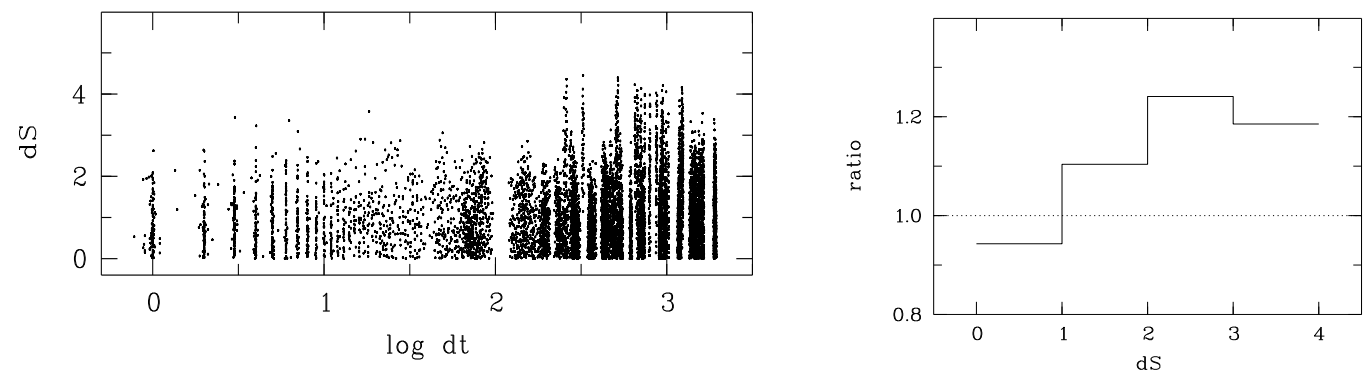

FIGURE 4. Left: dS(dt) distribution - differences in intensity between any two points, normalised to their significance. Right: Ratio of actual $\mathrm{dS}(\mathrm{dt}$ ) (for $\mathrm{dt} \sim 1$ day) for PKS $0528+134$ to those of the normal distribution for a constant source. There is a clear excess of differences at the 2-3 Sigma level, indicating variability on a statistical level. 


\section{Results and Discussion}

We computed structure functions of several gamma-bright Blazars using light curves obtained from maps with a temporal binning of one day. IDV is detected in the EGRET data on a significant level even during those observations when the sources are not exceptionally bright. Gamma-ray IDV has a high duty cycle and is not confined to individual flares. This is similar to the variability seen at lower photon energies, and permits cross-correlation studies even if IDV remains at low amplitudes. Comparing the fluxes of Blazars during their low states with those derived at fixed but "empty" positions in the same one-day maps, we find a statistical indication for PKS $0528+134$ and $0716+714$ to have a steady flux about 1 sigma above the background. This is consistent with previous upper limits but confirms that EGRET data are sensitive enough to reach even the lower envelope of the range in flux densities.

\section{REFERENCES}

1. Feigelson, E.D., Bradt, H., McClintock, J., et al. 1986. Ap.J., 302, 337-351.

2. Hartman, R.C., Bertsch, D.L., Dingus, B.L., et al. 1993. Ap.J.Lett, 407, L41.

3. Hartman, R.C., Webb, J.R., Marscher, A.P., et al. 1996. Ap.J., 461, 698.

4. Hunter, S.D., Bertsch, D.L., Dingus, B.L., et al. 1993. Ap.J., 409, 134-138.

5. Kendziora-Chudcer, L., Jauncey, D.L., Wieringa, M., Walker, M.A., Nicolson, G.D., et al., in IAU Coll. 164: Radio Emission from Galactic and Extragalactic Compact Sources, G. Taylor, J. Wrobel, \& A. Zensus (Eds.), 1997, in press.

6. Kniffen, D.A., Bertsch, D.L., Fichtel, C.E., et al. 1993. Ap.J., 411, 133-136.

7. Kraus, A., Ph.D. thesis, University of Bonn, 1997.

8. Mattox, J.R., Bertsch, D.L., Chiang, J., et al. 1993. Ap.J., 410, 609-614.

9. Mattox, J.R., Wagner, S.J., Malkan, M., et al. 1997. Ap.J., 476, 692.

10. Miller, H.R. \& Noble, J.C. 1996. in Blazar Continuum Variability, H.R. Miller, J.R. Webb, \& J.C. Noble (Eds.), ASP. Vol. 110, pp. 17-30.

11. Quirrenbach, A., Witzel, A., Qian, S.-J., et al. 1989. A.E A., 226, L1-L4

12. Remillard, R.A., Grossan, B., Bradt, H.V., et al. 1991. Nature, 350, 589-592.

13. Stein, W.A., in Pittsburgh Conference on BL Lac Objects, A.M. Wolfe (Ed.), Pittsburgh University, 1978, pp. 1-21.

14. Takalo, L.O., Sillanpää, A., Pursimo, T., et al. 1996, A.E A. Suppl., 120, 313.

15. Wagner, S.J. 1997. in IAU Coll. 164: Radio Emission from Galactic and Extragalactic Compact Sources, J. Wrobel, \& A. Zensus (Eds.), in press.

16. Wagner, S.J. \& Witzel, A. 1995. Ann. Rev. Astron. Astrophys., 33, 163-197.

17. Wagner, S.J., Mattox, J.R., Hopp, U., et al. 1995. Ap.J. Lett., 454, L97-L100.

18. Wagner, S.J., Witzel, A., Heidt, J., et al. 1996, A.J., 111, 2187-2211. 